\title{
OPHTHALMOLOGY
}

\section{Orbito-Ocular Teratoma: A Case Report}

\author{
E.A. Akabe, C.D. Mpyet and B.M. Mandong* \\ Departments of Ophthalmology and *Pathology, Jos University Teaching Hospital, Jos
}

\begin{abstract}
Orbital teratomas are rare. This is a report of a case of orbito-ocular teratoma with malignant transformation in a seventeen-day-old girl. Treatment was by exenteration. The baby was lost to follow-up shortly after" discharge and so could not have a prosthesis fitted ( Nig $J$ Surg Res 200(1; 2:155-157)
\end{abstract}

KEYWORDS: Orbito-ocular, teratomas, malignant transformation, exenteration

\section{Introduction}

Teratomas are neoplasms composed of the three germ layers of the embryo. They are the most common tumours of infancy and childhood.' ? Teratomas are most commonly found in the sacrocoxcygeal region, then the gonads, cervical and retroperitoneal regions of the body.' Teratomas arising from the head and neck region are uncommon. ${ }^{4}$ We report a case of orbito-ocular teratoma in an infant.

Case Report

$\Lambda$ seventeen day-old baby girl was referred from a Primary Ifealth Centre. The parents noticed a protruding mass in the left eye at birth, which had not changed in size but bled easily on contact and discharged pus. The child was a product of a term, uneventful but unsupervised pregnancy and was the fifth child of parents. All the other siblings were well and there was no known history of congenital abnormalities in the relations.

Examination showed a healthy looking girl, weighing $2.5 \mathrm{~kg}$. The left eyeball could not be seen except for a large, firm, fungating mass protruding from that socket. It measured $5 \mathrm{~cm} x$ $4 \mathrm{~cm}$; stretching both eyelids and having a mucopurulent discharge with crusted blood (figure 1). No regional lymph nodes were palpable. The right eye was normal. There were no abnormalities in other systems.

The haemogram, white cell and platelet counts were normal Skull X-ray showed slight enlargement of the left orbit. No calcifications were seen. Serum $\alpha$-fetoprotein assay and computer tomographic scan were not done due to lack of facilities.

At surgery (7days after admission), a firm mass arising from the eyeball was found. Only the posterior part of the sclera and the optic nerve could be seen. The mass, which appeared encapsulated, could easily be dissected from the tarsal conjunctiva and orbital periosteum allowing delivery enblock. A modified left orbital exenteration sparing the eyelids was performed.

Histology showed stratified squamous epithelium with keratinisation, cartilage, intestinal glands and neural tissue with retinoblasts showing severe anaplasia. There was local infiltration and foci of adipose tissue in addition to striated muscles. These findings

Reprint requests to: Dr. C.D. Mpyet, Department of Ophthalmology,Jos University Teaching Hospilal, P. M. B. 2076, Jos, Plateant State, - mail: mpletcónuijosedums 
were consistent with malignant transformation in a teratoma (Figure 2).

The patient was followed-up for only two weeks after discharge and was subsequently lost to follow-up. There was no opportunity for orbital reconstruction and artificial eye fitting.

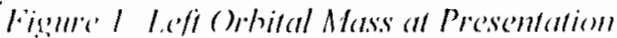

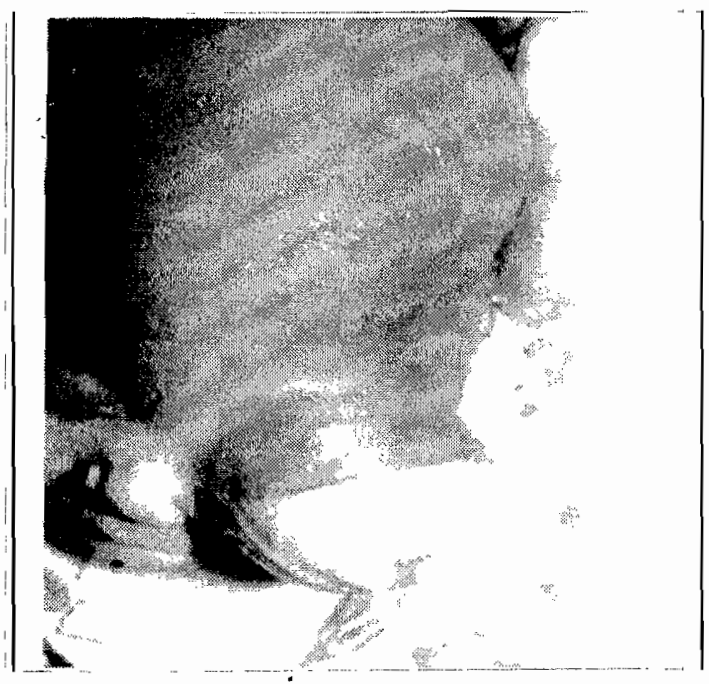

Higure 2: Histological Section Showing Matured Cartilage and Retinoblasts with Severe Anaplasia

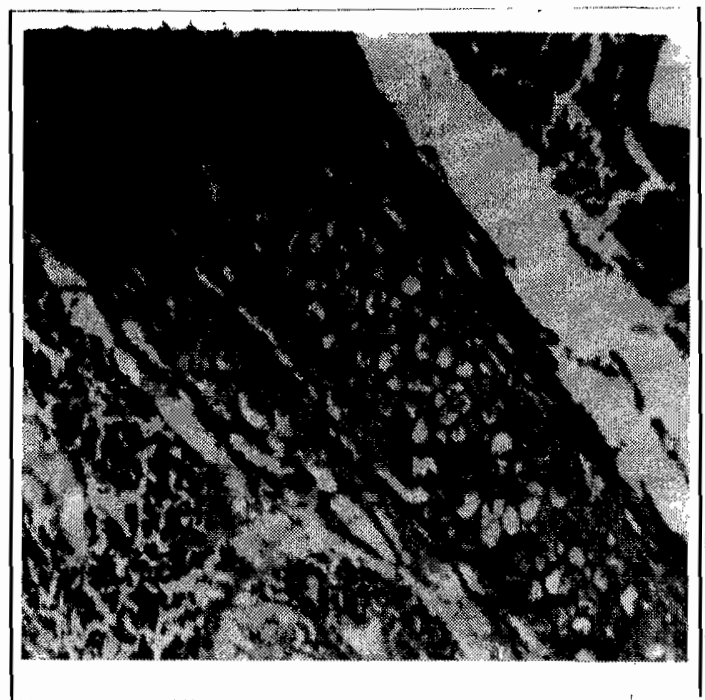

\section{Discussion}

Teratomas arising from the head and neck region are most commonly located in the anterior or lateral neck, followed by the face, mouth or nasopharynx and rarely from the orbit. ${ }^{2.5}$ This patient presented in the immediate newborn period and did not have other congenital anomalies; this is consistent with reports on head and neck teratomas. ${ }^{1.2}$

Facilities were not available for CT scan and orbital ultrasound, which are useful in determining the extent of the disease and differential diagnosis.

We had to rely on clinical evaluation and plain radiography to make a diagnosis. Potential for malignant transformation in teratomas arising from the eyeball as in other parts of the body has been reported.' There was malignant transformation in this patient.

Complete surgical excision through exenteration had to be undertaken to prevent the risk of recurrence. There was enlargement of the orbit and destruction of the eyeball as a result of pressure; this is consistent with other reports of pressure due to teratomas. ${ }^{1,6.7}$

This case of teratoma alludes to the biological behaviour of the tumour and the lact that teratomas are totipotential tumours that can arise from any part of the body especially in the midline structures. Teratomas have diverse anatomic locations and no organ can be said to be exempted. Careful histopathological examination of surgical specimens may reveal that the orbit is not an uncommon sitc.

\section{References}

1. Nimadu PT. Childhood teratoma in Zaria, Nigeria. East Afr Med J 1995; 72: 551553.

2. Taper D, Lack EE. Teratomas in infancy and childhood. A 54-year experience at the children's hospital Medical Centre. Ann Surg 1983; 198: 398-409.

3. Carr MM, Thomer P, Philips JH. Congenital teratomas of the head and neck. 
J Otolaryngol 1994; 26: 246-252.

4. Grosfeld JL, Balantine TVN, Lowe D, Borener RL. Benign and malignant teratomas in children: Analysis of 85 patients. Surgery 1976; 80;297-305.

5. Sharma MC, Sarkar C, Gaikwad S, Mahapatra AK, Bahadur S. Congenital orbital teratoma: A report of two cases. Indian J Ophthalmol 1977; 45:49-52.
6. Lee JT, Hall TR, Bateman JB. Optic nerve hypoplasia secondary to intracranial teratoma. Am J Ophthalmol 1997; 124: 705-706.

7. Lanzino G, Kaptain GJ, Jane JA, Lin KY. Successful excision of a large immature teratoma involving the cranial base: $A$ report of a case with long-term follow-up. Neurosurg 1998; 42: 389-393. 